\title{
Dynamic and Static Characteristics of Double Push Rods Electromechanical Converter
}

\author{
Xuping Wang ${ }^{1}$, Long Quan² ${ }^{*} \mathbb{D}$, Shiyi Luan ${ }^{2}$ and Xiaoqing Xu
}

\begin{abstract}
Due to the influence of material characteristics and winding power, single output electromagnet has limited ability to improve the dynamic characteristic of electro-hydraulic valve. Therefore, an electromechanical converter with double push rods is proposed in this paper, which can simultaneously output two electromagnetic forces, can push or pull the valve core and sleeve according to the current direction and realize rapid operation of load. According to the electromagnetic principle and the magnetic circuit analysis method, the mathematical model and equivalent circuit of the electromechanical converter with double push rods are established. Through the finite element simulation model of the electromechanical converter with double push rods with the same magnetization directions, the changing rules of its magnetic field distribution and force-displacement behaviors are studied and analyzed. According to the analysis results, the electromagnetic mechanical parameters and mechanical structure of the electromechanical converter with double push rods are determined, and the prototype is made. The test platform for the push-pull characteristics of the electromechanical converter with double push rods is built, and its static and dynamic characteristics are tested and analyzed. The results show that the thrust and pull output characteristics of the internal and external push rods are basically consistent with the simulation output, and proportional to the current density of the coil; the push-pull hysteresis of internal and external push rods output force is less than 5\%; and the dynamic time response characteristics of the displacement and force are obtained. The hysteresis effect of output force is improved effectively through the $\mathrm{H}$ bridge drive control circuit modulated by PWM. Compared with the displacement response of a singlewinding electromagnet with a similar volume, it can effectively improve the dynamic displacement response. Followup work will further optimize the structure of the electromechanical converter and test the corresponding pilot valve. The research results provide a new theory for improving the output characteristics of electro-hydraulic pilot valve and have an extremely high engineering application value and broad application prospect.
\end{abstract}

Keywords: Moving coil motor, Double push rods electromagnet, Proportional solenoid valve, Response characteristics

\section{Introduction}

In recent years, electro-hydraulic proportional valves have been widely used due to their simple structure, stable performance, high energy efficiency, and eco-friendliness [1-3]. With the development of automobile, aerospace and other industries, higher and higher demands are placed on the characteristics of their controlled valve body which is key control component, and response speed is one of the

\footnotetext{
*Correspondence: quanlong@tyut.edu.cn

${ }^{2}$ Institute of Mechanical Engineering, Taiyuan University of Technology, Taiyuan 030024, China

Full list of author information is available at the end of the article
}

important indicators. In industrial production, electromechanical converters are usually used to drive pilot valves or directly drive electro-hydraulic proportional valves. Their output characteristics determine the performance of proportional valves. The research conducted by scholars at home and abroad on electromechanical converter mainly focuses on the research of control strategy, the development of new electromechanical converters and the way to improve the response characteristics of electromagnetic valves. Among them, the response characteristics of electromagnetic valve are improved by control strategy. For example, Ahn developed a new PWM signal algorithm. Amirante et al. used the proposed boosted PWM 
technique to improve the open loop control technology $[4,5]$, which could effectively improve the response characteristics of electromagnetic valve. Refs. [6-9] analyzed and studied the current, turns per coil, PWM frequency, mechanical parameters and other key parameters of electromagnet influencing the output force of electromagnetic valve. Through observation experiments, Sun et al. [10] concluded that electromagnetic energy conversion was influenced by the rate of change of drive current. Too big current change would reduce the efficiency of conversion of electromagnetic energy into mechanical energy, thus accelerating power consumption. Thompson Corporation studied the structure and size parameters of the electromagnetic valve through a large number of experiments, and optimized the dynamic and static characteristics, volume and weight of the electromagnetic valve $[11,12]$. Other studies optimized structural design or the dynamic characteristics of electromagnetic valve by selecting soft magnetic materials with different B-H curves [13-16]. For example, Wang et al. [17] used AL-Fe material to prove by experiment that new soft magnetic material could significantly improve the performance of high-speed electromagnetic digital valve. Cheng et al. [18] designed the corresponding magnetic circuit based on the application of soft magnetic alloy material. Control rod hydraulic drive mechanism (CRHDM) of Institute of Nuclear and New Energy Technology, Tsinghua University innovates the mechanical structure. Its key part consists of three linear electromagnetic valves, so that it can obtain a large electromagnetic force in a short time [19]. In addition, new materials such as piezoelectric ceramics (PTZ) and giant magnetostrictive material (GMM) are selected. The electromechanical conversion element composed of PTZ has a high precision and a high response speed, but due to disadvantages such as relatively big hysteresis, the need to develop the corresponding compensation power supply, a small displacement and a high cost, currently the level of commercialization is not high $[20,21]$. Some studies use the electromagnetic parameters to estimate the displacement without the displacement sensor [22-25] or control valve with modern control method [26-28]. In order to improve the dynamic response characteristics of the displacement of the electromechanical converter, this paper proposed a new type of electromechanical converter with double push rods and analyzed and studied its dynamic and static characteristics.

\section{Working Principle and Mathematical Model of Magnetic Circuit of Double Push Rods Electromechanical Actuator}

\subsection{Quick Response Mechanism of Double Push Rods} Electromechanical Converter

The structure of the double push rods electromechanical converter proposed in this paper is shown in Figure 1.

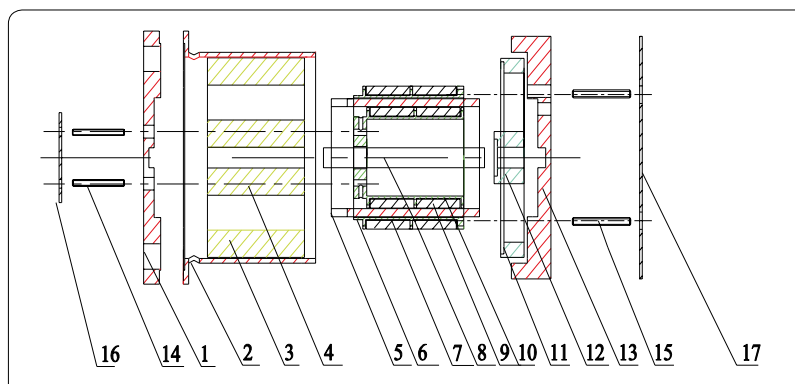

(a) Structure schematic diagram

1. Front cover; 2. Shell; 3. Outer permanent magnet; 4. Inner permanent magnet; 5 . Sleeve; 6 . Outer coil frame; 7. Outer coil; 8. Iron core; 9. Inner coil; 10. Inner coil frame; 11. Outer limit block; 12. Inner limit block; 13. Rear cover; 14. Inner rod; 15 . Outer rod; 16. Output unit; 17. Output unit

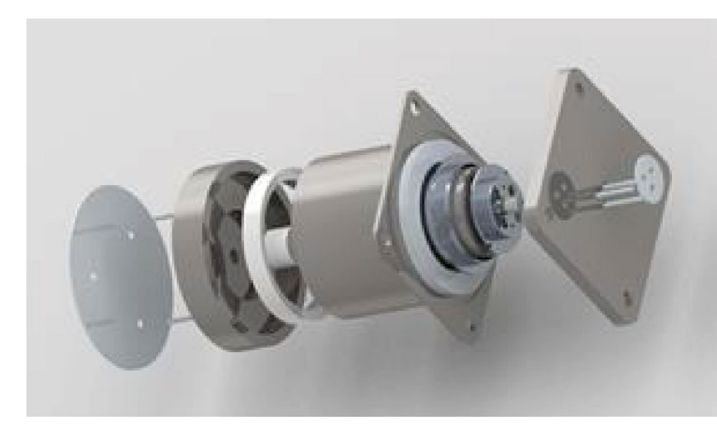

(b) Assemble sketch map

Figure 1 Double push rods electromechanical converter

It can output two electromagnetic forces at the same time. That is, it has two movable parts, which are the internal push rod and the external push rod. The internal push rod and the external push rod are respectively connected to the inner and outer coil racks. The current direction of two coils can generally be controlled, so that the internal push rod and the external push rod move in the same or reverse direction to control the valve flow. The external rod can also be fixed and the flow can be controlled by pushing the valve core through the internal rod. The internal rod can also be fixed and the flow can be controlled by controlling the valve bush through the external rod.

The rapid response mechanism is as shown in Figure 2. When the external rod and the valve sleeve controlled are not moving, the displacement response curve of the internal rod is $x_{\mathrm{a}}(t)$, the stable output flow value of the corresponding valve opening is $x_{\mathrm{v}}$, and the required time is $t_{\mathrm{a}}$, then $x_{\mathrm{a}}\left(t_{\mathrm{a}}\right)=x_{\mathrm{v}^{*}}$

On the contrary, when the internal rod is not moving, the displacement response curve of the external rod is $x_{\mathrm{b}}(t)$, the stable output flow of the corresponding valve opening is $-x_{\mathrm{v}}$, and the corresponding response time is $t_{\mathrm{b}}$, then $x_{\mathrm{b}}\left(t_{\mathrm{b}}\right)=-x_{\mathrm{v}}$. 


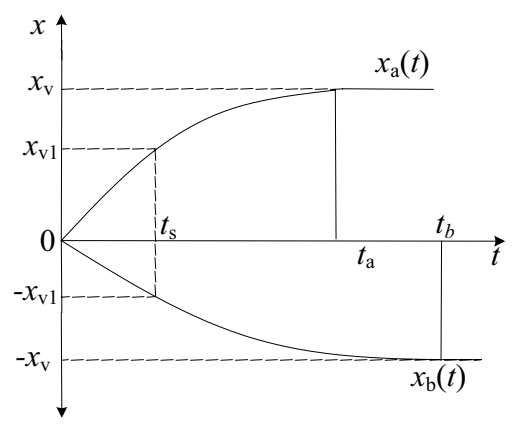

Figure 2 Quick response mechanism of the two-push-rods electromechanical converter

When both push rods respond at the same time, if the same stable flow of a single push rod is reached, there must be a time point $t_{\mathrm{s}}$, so that $x_{\mathrm{a}}\left(t_{\mathrm{s}}\right)-x_{\mathrm{b}}\left(t_{\mathrm{s}}\right)=x_{\mathrm{v}}$.

Obviously, $t_{\mathrm{s}}<t_{\mathrm{a}}$ and $t_{\mathrm{s}}<t_{\mathrm{b}}$. Therefore, when the valve opening is certain, the relative displacement of the internal and external push rods of the electromechanical converter can effectively reduce the response time and improve the displacement response [29].

\subsection{Magnetic Circuit and Mathematical Model}

In order to determine the electromagnetic and mechanical parameters of the double-rods electromechanical converter, equivalent magnetic circuit and finite element simulations of the double-push-rods electromechanical converter are analyzed and studied. The double push rods electromechanical converter is presented in Figure $1(\mathrm{a})$, in which the material of front cover 1 , rear cover 13 , shell 2 , sleeve 5 and iron core 8 is DT- 4 . The material of permanent magnet 3 and 4 is NeFe35. The intensity of magnetization is $\mathrm{N} 45$, and the magnetization direction is same. Outer coil frame 6 and inner coil frame 7 form the winding. The material of coil frames is aluminum alloy. The material of outer limit block 11 and inner limit block 12 is PTFE.

The design of magnetic circuit improves the utilization rate of permanent magnetic materials and reduces the loss of magnetic circuit. The permanent magnet adopts the radiated magnetization, and the structure of the electromechanical converter is a rotator around the central axis. NeFe35 is chosen as the permanent magnetic material, which has a large magnetic energy product and can effectively increase the electromagnetic force and reduce the size of the prototype. Figure 3 shows the equivalent electromagnetic circuit, in which $R_{\mathrm{m} 1}, R_{\mathrm{m} 2}, R_{\mathrm{m} 3}, R_{\mathrm{m} 5}$ $R_{\mathrm{m} 6}, R_{\mathrm{m} 8}, R_{\mathrm{m} 9}$ and $R_{\mathrm{m} 10}$ are the magnetic resistance of the different shells; $R_{\mathrm{n} 1}$ and $R_{\mathrm{n} 2}$ are the equivalent internal resistance of permanent magnet; $R_{\delta 1}$ and $R_{\delta 2}$ are the

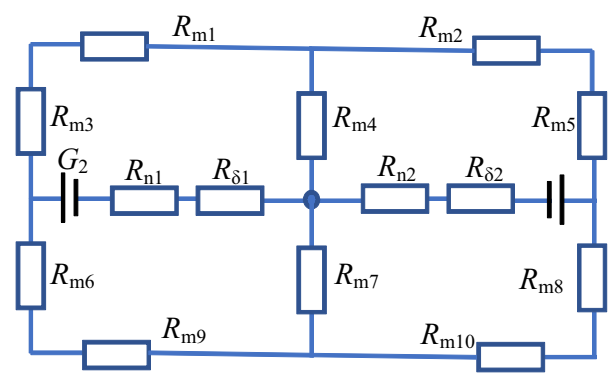

Figure 3 Equivalent circuit of two push-rods electromechanical converter

magnetic resistance of the working air gap; and $R_{\mathrm{m} 4}$ and $R_{\mathrm{m} 7}$ are the magnetic resistance of part 5 sleeve.

According to the equivalent magnetic circuit and the Kirchhoff's law of the magnetic circuit, the equation can be calculated as:

$$
G_{2}=\phi_{0}\left(R_{\delta}+R_{\mathrm{m}}+R_{\mathrm{n}}\right) .
$$

In which, Effective flux can be calculated:

$$
\phi_{0}=\sigma \phi_{\delta},
$$

where $G_{2}$ is the magnetic potential of the permanent magnet; $\Phi_{\delta}$ is the total magnetic flux of the permanent magnet; $R_{\delta}$ is the total air gap resistance; $R_{\mathrm{m}}$ is the total magnetic resistance of the magnetic material; $R_{\mathrm{n}}$ is total internal magnetic resistance of permanent magnetic material; $\sigma$ is leakage magnetic flux.

Formula for calculating the magnetic resistance of annular air gap and the internal resistance of permanent magnet:

$$
\begin{aligned}
& R_{\delta}=\frac{1}{2 u_{0} \pi h}\left(\ln r_{1}-\ln r_{2}\right), \\
& R_{\mathrm{m}}=\frac{1}{2 u_{\mathrm{m}} \pi h_{1}}\left(\ln r_{3}-\ln r_{4}\right) . \\
& \phi_{0}=B S .
\end{aligned}
$$

In which $h_{1}$ is the thickness of magnetic material; $r_{3}, r_{4}$ are the outer diameter and inner diameter of magnetic material; $u_{0}$ is the vacuum permeability; $h$ is working air gap length; $r_{1}, r_{2}$ are outer diameter and inner diameter of cylindrical air gap; $u_{\mathrm{m}}$ is magnetic permeability of magnetic material; $B$ is magnetic induction intensity of working magnetic field; $S$ is cross-sectional area of working magnetic field; $r_{5}$ is the inner diameter of the permanent magnet.

The electromagnetic force of the inner rod:

$$
F=\frac{G_{2} i L \sigma}{\left[\frac{1}{\mu_{0} h}\left(\ln r_{1}-\ln r_{2}\right)+\frac{1}{\mu_{\mathrm{n}} h}\left(\ln r_{2}-\ln r_{5}\right)\left(r_{3}-r_{4}\right)^{2}\right]} .
$$


Through the above formula, according to the electromagnet volume and the experience design value of magnetic and electric load, the main electromagnetic and mechanical parameters of electromagnet can be obtained through analysis and calculation of magnetic circuit.

\subsection{Finite Element Model of Double Push Rods Electromechanical Converter}

Compared with the results of the magnetic circuit calculation, the results of the finite element calculation are more accurate. The basic electromagnetic and mechanical parameters are determined based on magnetic circuit analysis. By using Ansoft finite element analysis, the electromagnetic properties of the electromechanical converter with two push-rods are obtained by modeling, setting materials, grid division and post-processing.

Axisymmetric column coordinates in the two-dimensional modeling are selected. The coil working stroke length, between front end cover and back end cover is 2 $\mathrm{mm}$, respectively, so as to determine the zero position. The finite element mesh model of electromagnet with double push rods is shown in Figure 4. The maximum side length of the grid division unit is $3 \mathrm{~mm}$, and some errors are no more than $0.1 \%$. The boundary conditions are given. The outer boundary are the balloon boundary conditions, in the middle are the singular symmetric boundary conditions. After input of specific data: material properties, grid division and others are added. The main parameters are shown in Table 1.

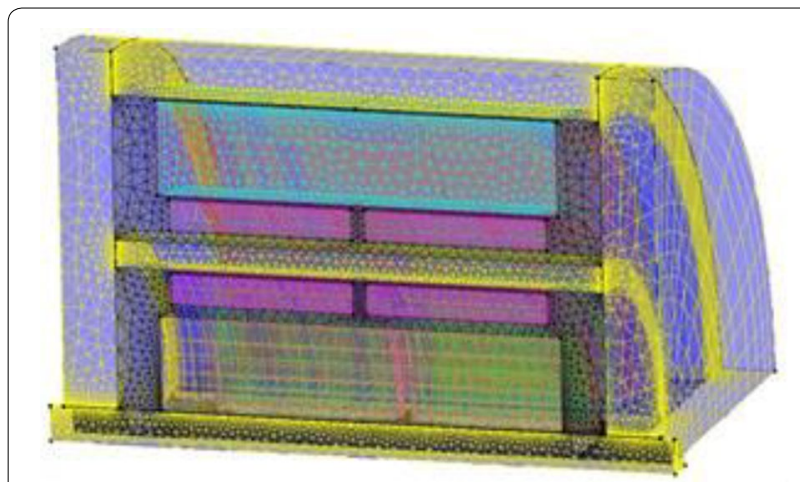

Figure 4 Meshing diagram of double rods electromechanical converter

Table 1 Main parameters

\begin{tabular}{ll}
\hline Boundary conditions & Zaxis: symmetry (odd) \\
& The other three sides: balloon \\
Incentive source & DC excitation \\
Solving parameters & Force of winding and rod \\
Meshing & Maximum side length $6 \mathrm{~mm}$ \\
Material & Shell: iron; coil: copper; rod: PTFE \\
& Permanent magnet: NdFe35 \\
\hline
\end{tabular}

The magnetic flux leakage is that the magnetic source leaks through the specific magnetic circuit in the space. The magnetic flux leakage coefficient can be calculated accurately by using finite element simulation.

The flux density results of ferromagnetic material and air gap is in a reasonable range. Figure 5 is the flux density cloud diagram in which the internal and external permanent magnet has the same direction of magnetization. The magnetic circuit is designed to offset the magnetic circuit of the middle sleeve, which can help to reduce the flux density of sleeve 5 to an extremely low value. The thickness of sleeve 5 can be appropriately reduced, and the number of effective coils can be increased, so as to optimize the size and quality of the electromechanical converter. The effective stroke of the inner and external push rod is $4 \mathrm{~mm}$. Figure 6 shows the electromagnetic force-displacement

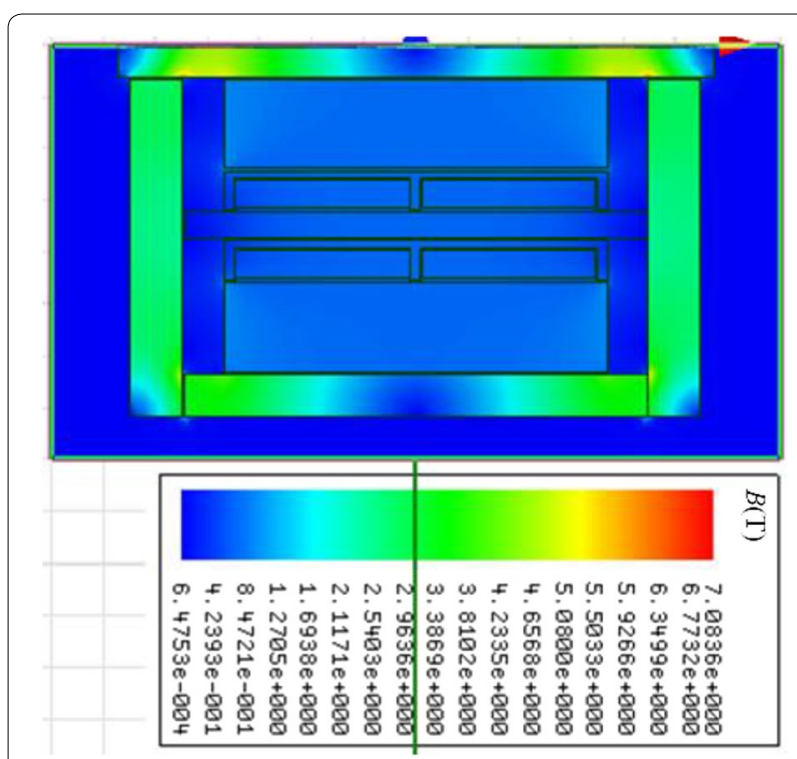

Figure 5 Magnetic field intensity with the same magnetization direction

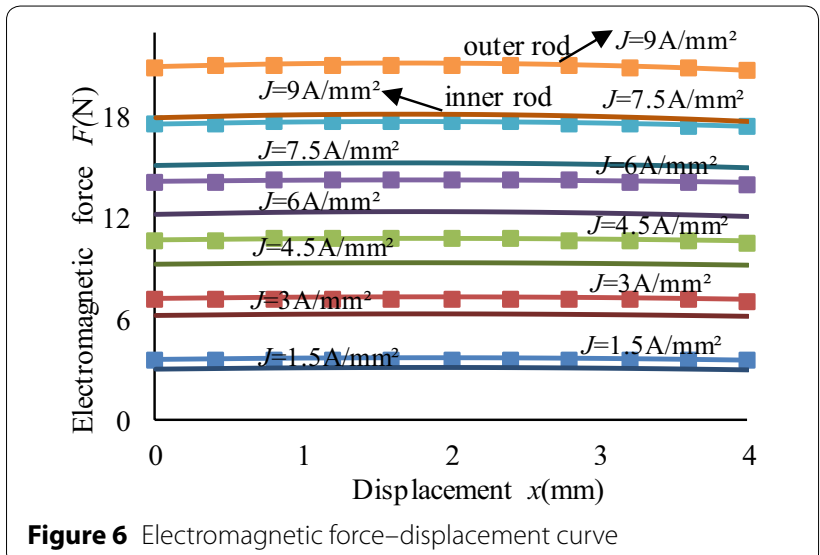


characteristics under different current densities. Seen from the figure, in the whole stroke the electromagnetic force output is basically constant. The higher the current density is, the greater the output force is.

The electromagnetic force on both sides of the stroke decreases because there are many leakage magnetic fields. The electromagnetic force output by the external push rod is similar to that of the internal push rod.

\section{Testing Device and Results of Double Rods Electromechanical Converter}

\subsection{Testing Device}

Figure 1 is the structure diagram of the double push-rods electromechanical converter. In fact, considering size limit of machine tool, thus chamfering of locating slot is less than $0.5 \mathrm{~mm}$; The outer cover 2 , the sleeve 5 , the core 8 at the joint with the front and rear ends have a chamfer of less than $0.5 \mathrm{~mm}$; and the above parts have wire holes in specific positions for out of the wire.

Considering economic performance and installation, the permanent magnet 3 and 4 , each is divided into 4 pieces (less than 90 degrees each) that assembly process is equipped to facilitate heat dissipation of the coil and satisfy the wet solenoid valve. Because the outer frame 6 and the inner frame 10 are made of aluminum alloy with a high hardness, which may scratch the enameled coil, the coil groove is chamfered. The function of the outer limiting block 11 and the inner limiting block 12 which are made of PTFE is to position the permanent magnet during installation. In order to ensure sealing, a groove is designed on the limiting block for adding a sealing ring.

The test apparatus of electromagnet with double pushrods is as shown in Figure 7. The test equipment includes an electromechanical converter, positioning devices, sensors, a display instrument and an acquisition system. Force sensors are fixed on the sliding platform and connected to

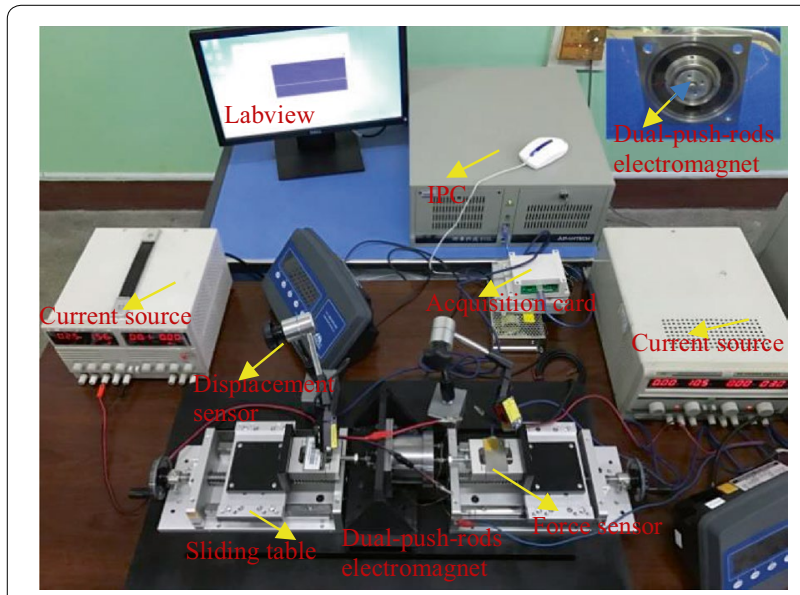

Figure 7 Test devices of double rod electromechanical converter the tested device, namely the electromechanical converter with double push rods. The stress and tension of the electromechanical converter can be measured through the selflock of the nuts. The laser displacement sensor positions through the magnetometer base. The data acquisition system consists of hardware and software. The hardware is the numerical indicator and acquisition card of the force sensor, which communicates with the upper computer.

\subsection{Experiment Results}

\subsubsection{Static Characteristic}

The winding is respectively fed with corresponding current, the internal rod outputs pressure and pushes the valve core, the external rod outputs pull and pulls the valve sleeve, and the electromagnetic valve is opened. Reverse current is input into the winding, the internal rod outputs pull and pulls the valve core, the external rod outputs pressure and pushes the valve bush, and the electromagnetic valve is closed. Therefore, Spool and sleeve simultaneously move when the internal and external rods are simultaneously actuated. By adjusting the moving coil displacement, the static characteristics of the displacement and thrust-pull force of the external and internal rods can be obtained, as shown in Figure 8 and Figure 9.

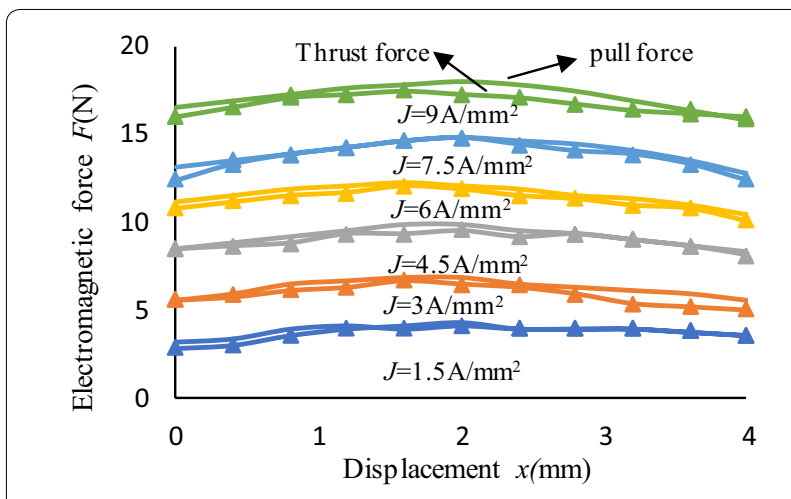

Figure 8 Electromagnetic force-displacement curve of inner rod

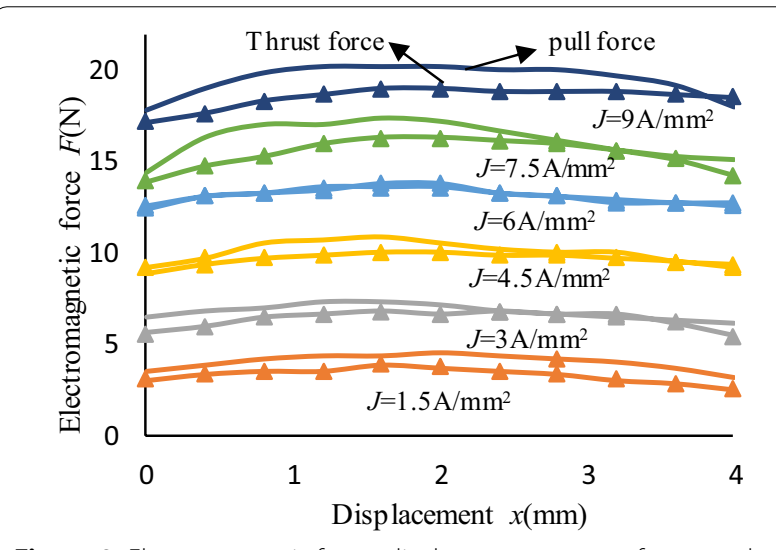

Figure 9 Electromagnetic force-displacement curves of outer rod 
The curve shown in Figure 8 is a set of displacementthrust and pull force curves under different current densities of the internal rod. The curve is similar to the simulation results, and the output force at the edge of the permanent magnet is slightly lower than that in other air gap positions. The output force in the effective stroke is basically constant. The electromagnetic force increases in proportion to the input current. The maximum pull appears when the air gap is around $1.6 \mathrm{~mm}$. At the current density of $9 \mathrm{~A} / \mathrm{mm}^{2}$, the pull is around $18 \mathrm{~N}$. The curves shown in Figure 9 show the displacement-push and pull force characteristics of the external rod, which are similar to those of the external rod. The maximum output force current density is $9 \mathrm{~A} / \mathrm{mm}^{2}$ and the pull is $15 \mathrm{~N}$. The direction of the electromagnetic force output by the internal and external rods is determined by the current direction. When the electromagnetic force is required to increase, the current density of the coil can be increased or the mechanical size of the electromagnet can be increased. It can be seen from the push-pull force characteristic curves of the rods that the frictional force is basically the same when the moving coil is running in the opposite direction.

\subsubsection{Hysteresis Characteristics}

Because of the friction of moving coil and the inevitable hysteresis of ferromagnetic materials, the output force will have a certain degree of hysteresis. The hysteresis can be corrected by a certain flutter current or reduced friction, so as to it can meet the engineering application requirements. Figure 10 and Figure 11 show the hysteresis curves of the pull and thrust force of the inner rod.

Figure 10 shows the current density-thrust force hysteresis characteristics of the inner rod at the working stroke of $1 \mathrm{~mm}, 2 \mathrm{~mm}$ and $3 \mathrm{~mm}$, respectively, with a force hysteresis degree of $3.2 \%$ (less than 5\%). Figure 11 shows the current density-pull force hysteresis characteristics of the inner rod at the working stroke of $1 \mathrm{~mm}$,

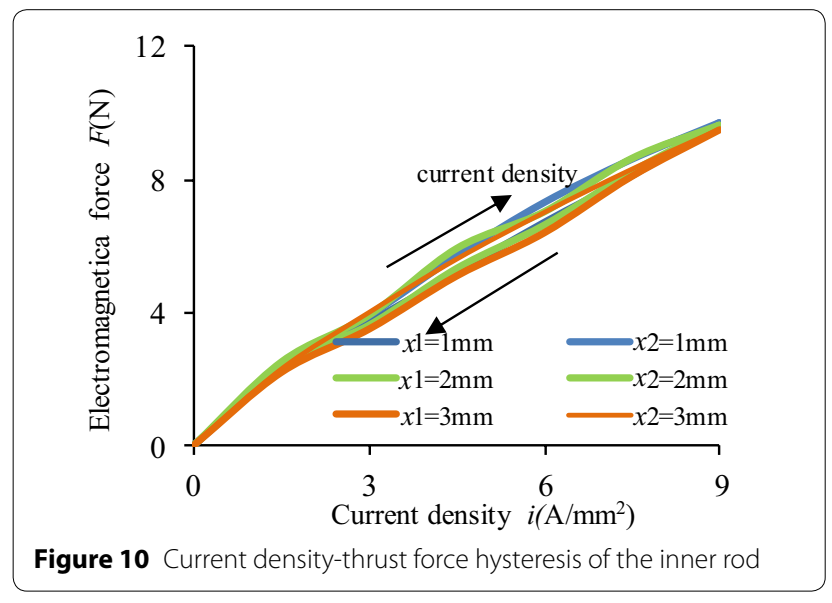

$2 \mathrm{~mm}$ and $3 \mathrm{~mm}$, respectively, with a force hysteresis degree of $4.9 \%$ (less than $5 \%$ ).

When the pilot valve is normally driven by the electromechanical converter, the inner and outer coils are driven by the PWM modulating amplifier. The frequency of PWM carrier signal can change from $200 \mathrm{~Hz}$ to $2000 \mathrm{~Hz}$. As a flutter signal, the carrier signal can improve the hysteresis of the output force. The drive circuit is $\mathrm{H}$ bridge, and the winding current signal is obtained by sampling resistance. The control principle is shown in Figure 12. When the carrier frequency works in a low range (around $200 \mathrm{~Hz}$ ), the coil vibrates more, and the carrier frequency is regulated to improve the output characteristics of the moving coil.

The output characteristics of the pull hysteresis of the internal push rod are improved significantly after being driven by an amplifier, as shown in Figure 13. The hysteresis has decreased to $0.6 \%$ and below. The experimental results show that the carrier signal can effectively improve the hysteretic effect.

\subsubsection{Dynamic Characteristics of Electromagnetic Force}

The dynamic characteristics of the electromagnetic force are determined by the efficiency of converting input
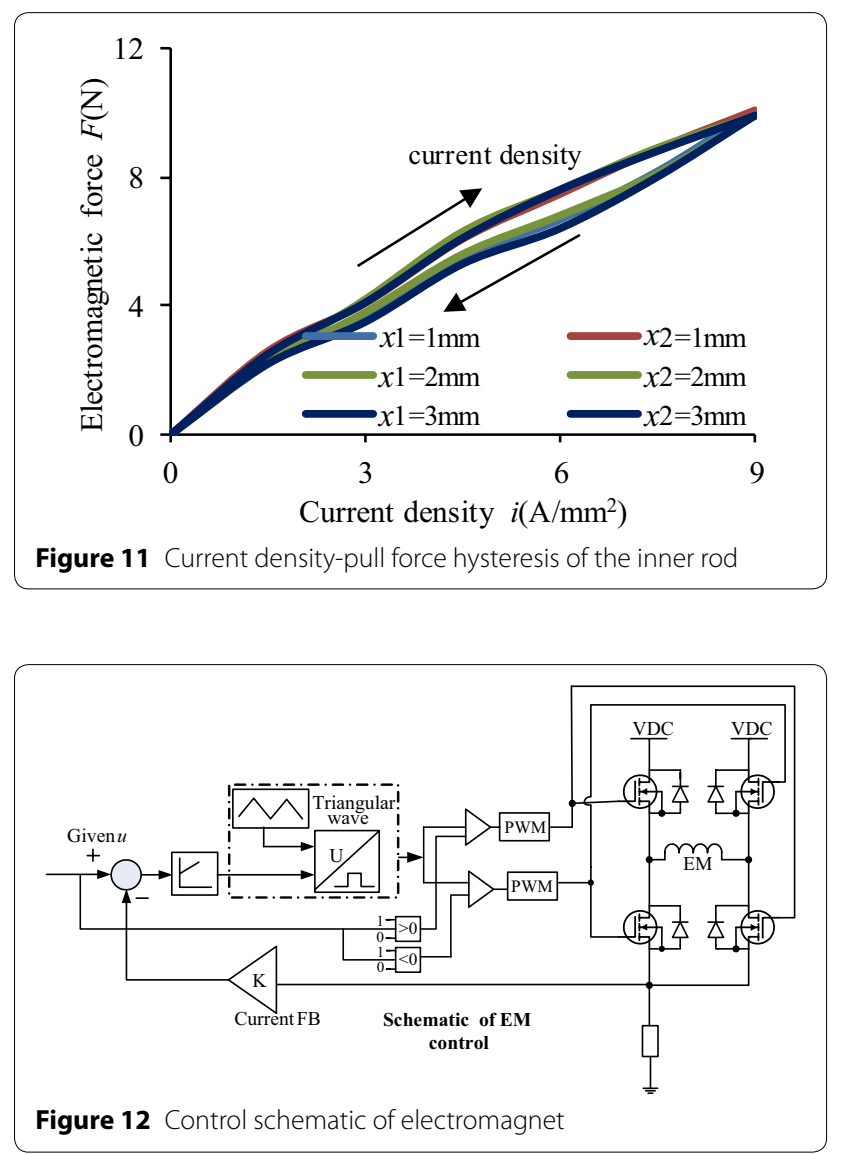


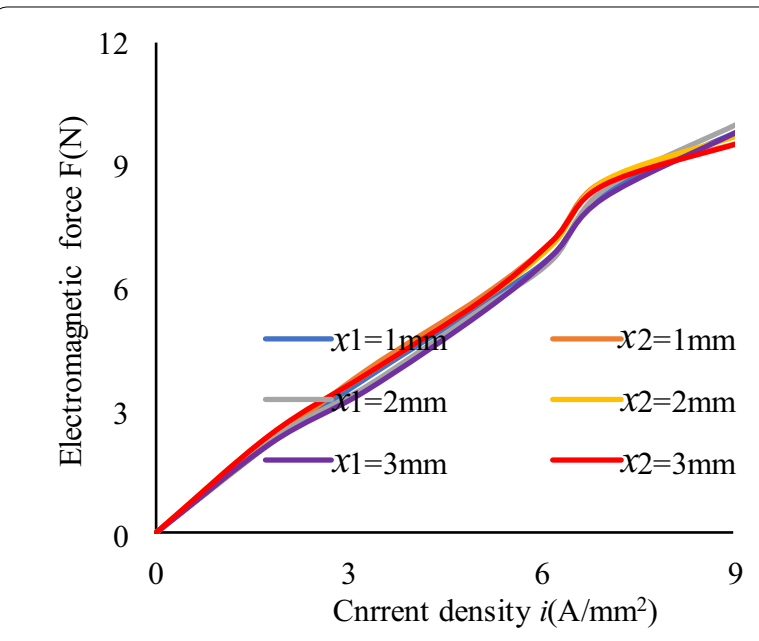

Figure 13 Electromagnetic force output by PWM driving

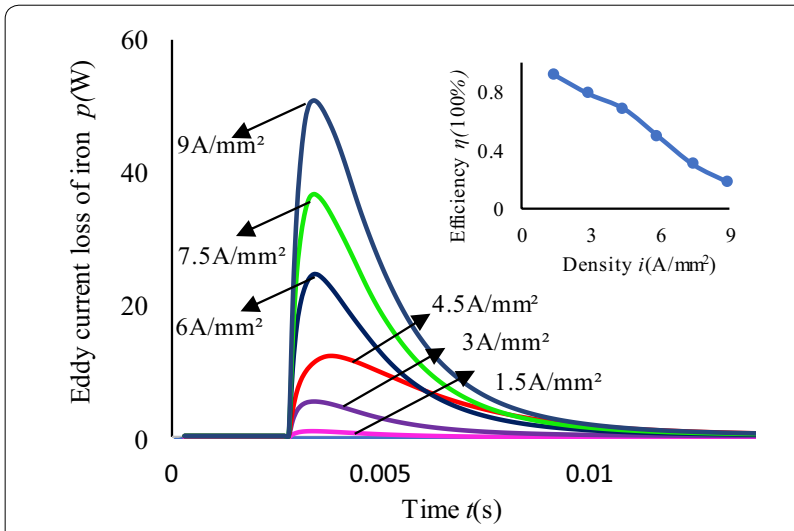

Figure 14 Eddy current loss and power conversion efficiency of iron core

power into mechanical power. The smaller the power loss is, the higher the conversion efficiency is, the faster the dynamic response time of the force is. In the process of step excitation for winding, input power $P_{1}=U I$, which is converted to mechanical power and loss power. The loss power includes copper consumptions, eddy current loss and hysteresis loss of iron core, and eddy current loss and hysteresis loss of permanent magnet. The copper consumption of the coil is relatively small, which can be ignored. The eddy current loss of permanent magnets and ferromagnetic materials accounts for the vast majority of the total loss, the eddy current loss of ferromagnetic materials is far more than permanent magnet eddy current loss. The magnetic hysteresis of the magnetic material is less than $5 \%$, which is negligible.

Figure 14 shows the finite element simulation results of the iron core eddy current loss under different current

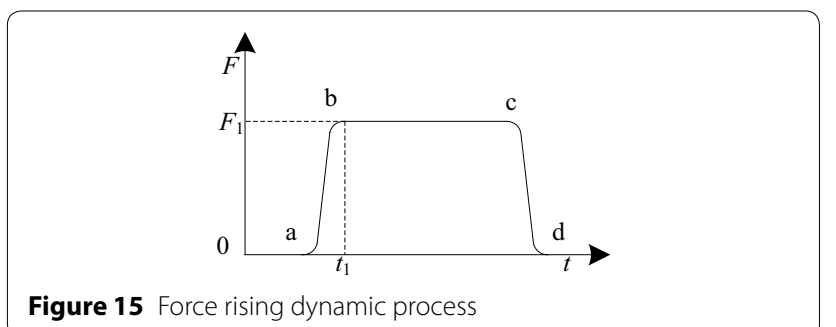

Figure 15 Force rising dynamic process

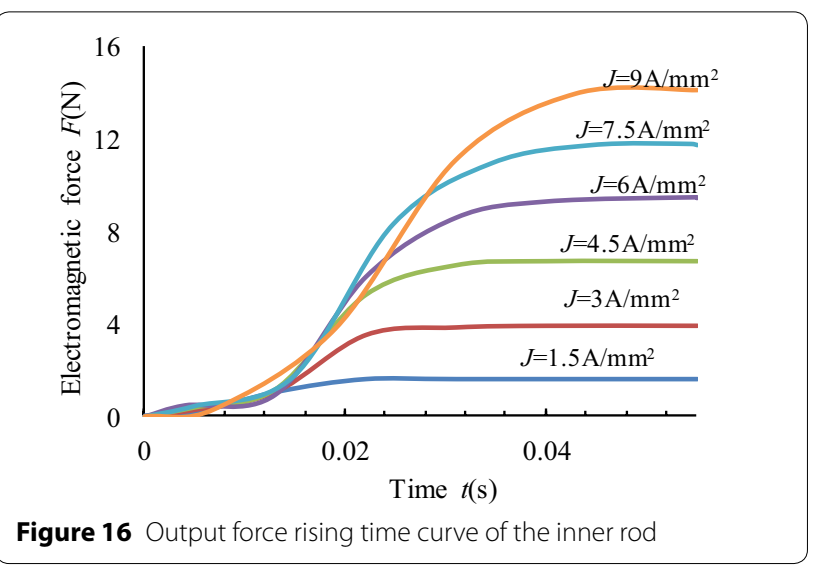

and the efficiency of the input power converting into effective power during the input excitation of the electromagnet. As can be seen, the efficiency rate of change in the $1-5 \mathrm{~A} / \mathrm{mm}^{2}$ range with a relatively small current density is relatively slow. With the increase of input current density, the loss of iron core increases and the efficiency decreases faster.

The electromagnetic force rising dynamic process is shown in Figure 15, in a single experiment, the coil does not move, at ' $a$ ' point coils input current, to reach the ' $b$ ' point corresponding to the force stability value, 'c' point releases current, the force is 0 at ' $\mathrm{d}$ ' point. $t_{1}$ is the corresponding response time. A set of curves between response time of force, displacement and current density is obtained.

Figure 16 shows the electromagnetic force rising time curve of the inner rod when the moving coil displace is $1 \mathrm{~mm}$ and the current density changes from $1.5 \mathrm{~A} /$ $\mathrm{mm}^{2}$ to $9 \mathrm{~A} / \mathrm{mm}^{2}$. It can be seen that with the increase of current step the response time of the electromagnetic force is longer, and the trend is that the difference in the adjacent current density response time is larger. The maximum response time is about $40 \mathrm{~ms}$ when the input step current is $9 \mathrm{~A} / \mathrm{mm}^{2}$. The higher the step of the input current in the same time is, the bigger the rate of current change is, the greater the influence of eddy current in the iron core is, the lower the power conversion 
efficiency is, the slower the increase of electromagnetic force is. Therefore, as the current step value increases, the response time will increase correspondingly. It is not a linear increase judging from the test results.

Figure 17 shows the force time response curve of the external push rod, which is similar to that of the internal rod.

Figure 18 shows the force response time curve of the output force when the current density varies from $1.5 \mathrm{~A} /$ $\mathrm{mm}^{2}$ to $9 \mathrm{~A} / \mathrm{mm}^{2}$ under different displacement. When the current density rises gradually from $1.5 \mathrm{~A} / \mathrm{mm}^{2}$ to $9 \mathrm{~A} / \mathrm{mm}^{2}$, the time for the electromagnetic force of the inner push rod to reach stable value gradually increases, but not linearly.

The basic trend is that the force response time is relatively stable when the current density is $1.5 \mathrm{~A} / \mathrm{mm}^{2}$ to $5 \mathrm{~A} / \mathrm{mm}^{2}$, and the slope of rising time is small. When the step current density is greater than $5 \mathrm{~A} / \mathrm{mm}^{2}$, the response time rises rapidly. The reason is that the induced potential and eddy current due to the step current is relatively large.

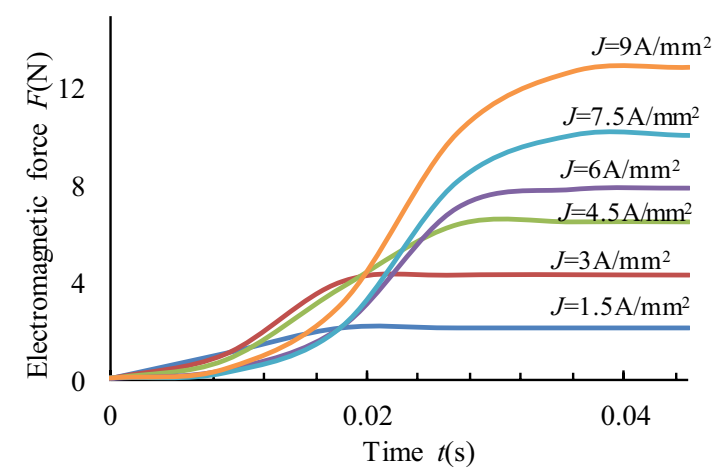

Figure 17 Output force rising time curve of the outer rod

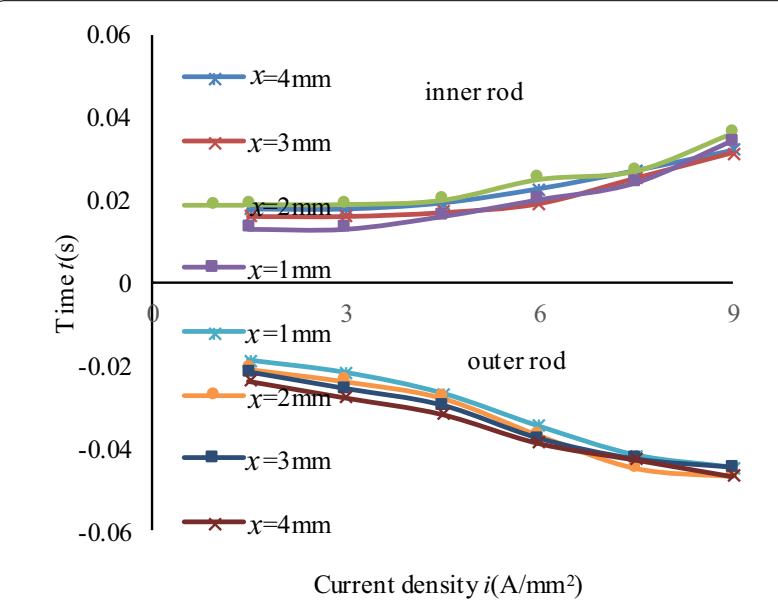

Figure 18 Force response time curve of inner and outer rod
When the winding of inner rod arrives at the $4 \mathrm{~mm}$ position and the current density is $9 \mathrm{~A} / \mathrm{mm}^{2}$, the response time is the longest, reaching about $0.04 \mathrm{~s}$. Therefore, it is necessary to study the relationship between the electromagnet parameters and the force response time, and to optimize the output characteristics of the electromagnet. The force response time curve of the output rod is similar with the time of the inner rod.

\subsubsection{Displacement Response Characteristics of No-Load}

The displacement dynamic characteristic curves of the electromechanical converter under different working conditions are the key of the working performance of the electromagnetic valve.

Figure 19 shows the displacement dynamic working curve. Point ' $a$ ' is the starting point of the operation process when the current input into coil. Then, coil moves to the displacement of point ' $\mathrm{b}$ '. The time $t_{1}$ between point ' $a$ ' and point ' $b$ ' is defined as stable displacement time. Reverse current is applied at point ' $c$ ' and return to zero at point 'd'.

Figure 20 shows that the displacement response time curves of inner and outer rod at the working stroke of 1 $\mathrm{mm}$ under different current densities. With the winding current density increasing, the response time decreases

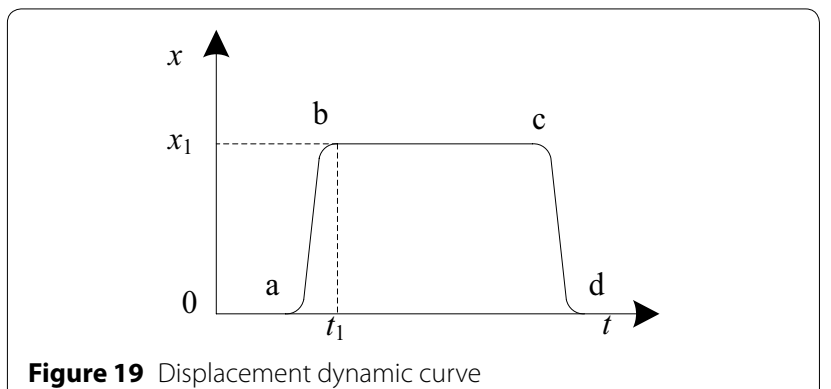

Figure 19 Displacement dynamic curve

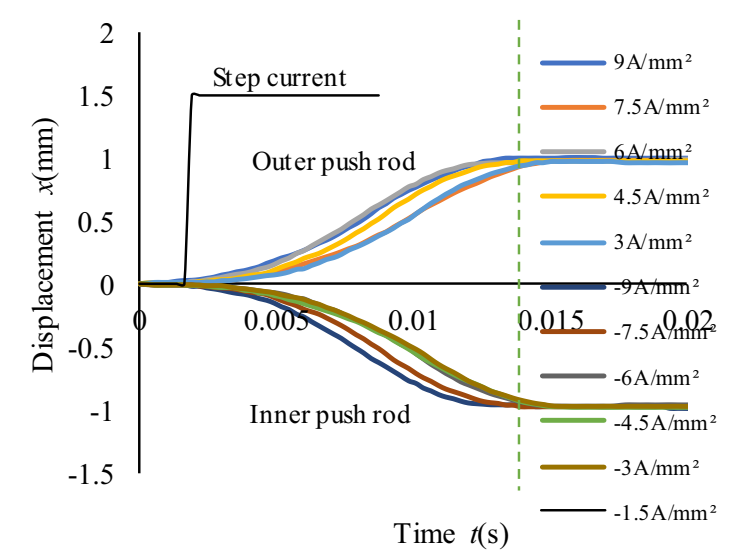

Figure 20 Displacement response curve of inner and outer rods 
gradually. The difference in displacement response time of the adjacent current density is reduced gradually. When the current density is $9 \mathrm{~A} / \mathrm{mm}^{2}$, the stable response time is about $9 \mathrm{~ms}$. This response time is related to the electromagnetic force and the number of turns.

Figure 21 shows that the displacement response time characteristics curves of the outer rod and inter rod under different working stroke. With the current density increasing from $1.5 \mathrm{~A} / \mathrm{mm}^{2}$ to $9 \mathrm{~A} / \mathrm{mm}^{2}$, the displacement response time decreases gradually, but the reduction rate decreases gradually, which demonstrates that current density effect on electromechanical valve gradually decreases. Especially when the working stroke is $1 \mathrm{~mm}$ and $2 \mathrm{~mm}$, and the current density changes from $4.5 \mathrm{~A} / \mathrm{mm}^{2}$ to $9 \mathrm{~A} / \mathrm{mm}^{2}$, the displacement response time reduction rate is less than $5 \%$. With the current density increasing proportionally, the efficiency of electromagnetic energy transforming into mechanical energy was getting lower and lower. As shown in Figure 21, in the range of current density less than $5 \mathrm{~A} / \mathrm{mm}^{2}$, the increase of current density helps to improve the dynamic response. However, when the current density exceeds $5 \mathrm{~A} / \mathrm{mm}^{2}$, the increase of the current density is not obvious to the improvement of the dynamic response time [30]. The displacement response time curve of the outerrod is similar to that of the inner-rod.

It can be seen from Figures 20 and 21 that the displacement response time of the inner and outer coils under the same current is similar.

In order to compare the displacement response of single-winding electromagnet, a single-winding electromechanical converter with a similar volume is selected. The test platform is shown in Figure 22 below. The volumes are $(10 \times 6 \times 6) \mathrm{cm}^{3}$ and $\left(\pi(7.6 / 2)^{2} \times 6.5\right) \mathrm{cm}^{3}$, respectively.

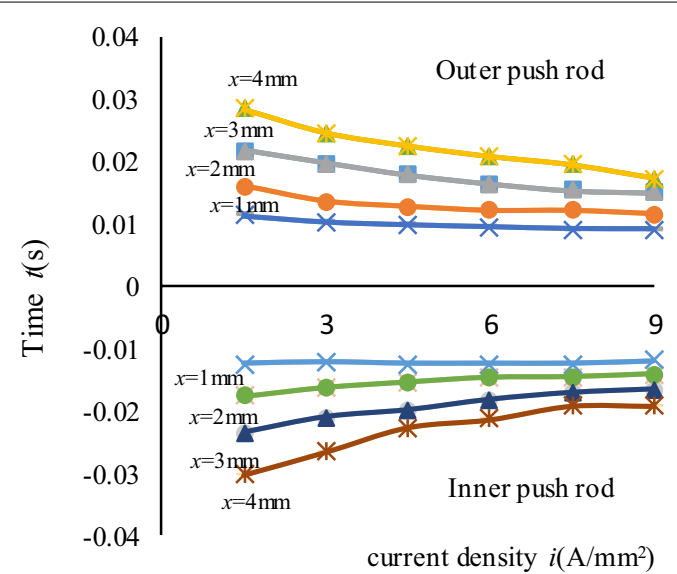

Figure 21 Displacement response time curve of the outer rod and inter rod

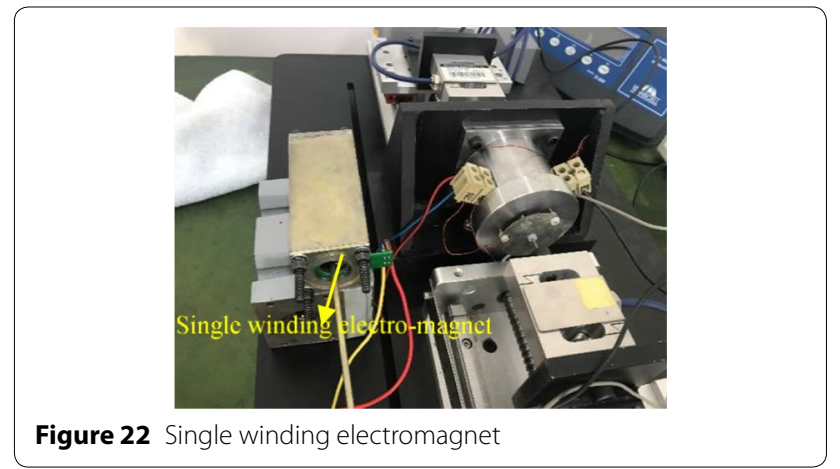

When the same current of the electromechanical converter with double rods is applied to the coil of the single-winding electromechanical converter, its displacement response curve is shown in Figure 23 below. The time for reaching $1 \mathrm{~mm}$ is about $10 \mathrm{~ms}$. The time of reaching $4 \mathrm{~mm}$ displacement varies from $25 \mathrm{~ms}$ to $36 \mathrm{~ms}$ under input current 3-9 $\mathrm{A} / \mathrm{mm}^{2}$. It can be seen from the figure that the response time of the electromechanical converter with double push rods and the single-winding electromechanical converter is similar and about $36 \mathrm{~ms}$ under the same current at the displacement of $4 \mathrm{~mm}$.

However, when the inner and outer coils of the double-rods electromechanical converter add the same current at the same time, as shown in Figure 24, the internal and external coils move each $2 \mathrm{~mm}$ in opposite directions respectively, the time of relative displacement of $4 \mathrm{~mm}$ can be reduced to about $20 \mathrm{~ms}$. Therefore, the displacement response time can be improved effectively by the electromechanical converter with double push rods.

The subsequent work will focus on the structural optimization and improvement of controller characteristics. The mechanical size and winding parameters will be further optimized, and the characteristics of the

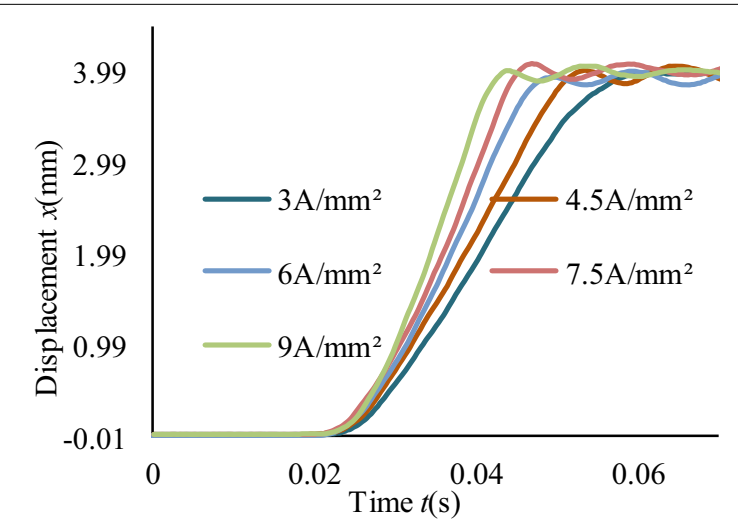

Figure 23 Displacement response of single winding electromagnet 


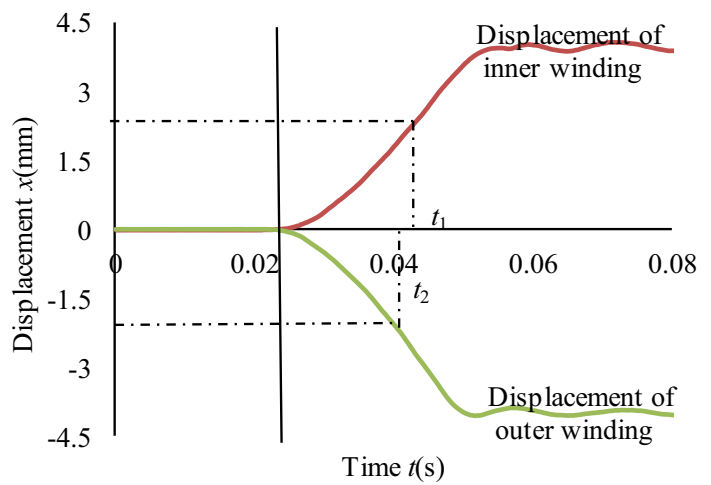

Figure 24 Rapid response of double push rods electromagnet

electromagnetic valve with double degrees of freedom will be analyzed and studied.

\section{Conclusions}

It is proposed a new structure double push-rods electromechanical converter, which can simultaneously output two same direction or reverse electromagnetic forces, and the dynamic and static output characteristics of the electromechanical converter were analyzed.

(1) A mathematical model of the electromechanical converter with double push-rods was established and the finite element simulation was conducted to determine electromagnetic mechanical parameters.

(2) In this paper, a prototype of double push-rods electromechanical converter was constructed, and a test platform for measuring two-way output force was set up. PWM-modulated H-bridge driving improved the hysteresis characteristics of output force.

(3) The dynamic and static characteristics of the double push-rods electromechanical converter was studied respectively, the electromechanical converter can output linear electromagnetic force, and can effectively enhance the speed of dynamic response of the control object. It was compared with the singlewinding electromagnet with a similar volume.

This study provides a new theory for improving the output characteristics of the electro-hydraulic proportional valve, which has very high engineering application value and broad application prospect.

\section{Authors' Contributions}

XW was in charge of the whole trial; XW and SL wrote the manuscript; XW, SL built test-bed, XW and XX assisted with sampling and laboratory analyses. LQ directs the whole project. All authors read and approved the final manuscript.

\section{Authors' Information}

Xuping Wang, born in 1976, is currently an associate professor at College of Electrical Power, Taiyuan University of Technology, China. She received her PhD degree from Taiyuan University of Technology, China, in 2014. Her research interests include the electro-hydraulic servo system components, control theory and methods, special motor.

Long Quan, born in 1959, is currently a professor and a PhD candidate supervisor at Institute of Mechatronics Engineering, Taiyuan University of Technology, China. His research interests include mechatronics engineering, fluid power transmission and control.

Shiyi Luan, male, born in 1994, is currently a postgraduate at Institute of Mechatronics Engineering, Taiyuan University of Technology, China. His research interests include mechatronics engineering, fluid power transmission and control.

Xiaoqing Xu, born in 1965, is currently an associate professor at Institute of Mechatronics Engineering, Taiyuan University of Technology, China.

\section{Acknowledgements}

The authors sincerely thanks to Professor Guangyu Xiong of Taiyuan University of Technology for his critical discussion and reading during manuscript preparation.

\section{Competing Interests}

The authors declare that they have no competing interests.

\section{Funding}

Supported by National Natural Science Foundation for Young Scientists of China (Grant No. 51505317), and Shanxi Provincial Natural Science Foundation of China (Grant No. 201601D102039)

\section{Author Details}

${ }^{1}$ College of Electrical and Power Engineering, Taiyuan University of Technology, Taiyuan 030024, China. ${ }^{2}$ Institute of Mechanical Engineering, Taiyuan University of Technology, Taiyuan 030024, China.

Received: 13 May 2018 Revised: 2 December 2018 Accepted: 10 July 2019 Published online: 19 July 2019

\section{References}

[1] Jian Hu, Wutian Wu, Shen Yuan, et al. Opening and closing of a novel high-speed switching valve. Proceedings of the Institution of Mechanical Engineers, Part I: Journal of Systems and Control Engineering, 2011, 226 (4): 466-475.

[2] Qianfeng Liu, Hanliang Bo, Benke Qin. Analysis of electromagnetic field of direct action solenoid valve with structure changing. Atomic Energy Science and Technology, 2008, 42 (1): 689-693.

[3] A D Gaeta, L Glielmo, V Giglio et al. Modeling of an electromechanical engine valve actuator based on a hybrid analytical FEM approach. IEEE/ ASME Transactions on Mechatronics, 2008,13(6): 625-637.

[4] R Amirante, L A Catalano. Boosted PWM open loop control of hydraulic proportional valves. Energy Conversion and Management, 2008, 49(2008): 2225-2336.

[5] Ahn Kyoungkwan, Yokota Shinichi. Intelligent switching control of pneumatic actuator using on/off solenoid valves. Mechatronics, 2005, 15(6): 683-702.

[6] ChunQing Li, Feng Jia, Minghai Li, et al. Research on dynamic response characteristics of high- speed solenoid valve. Computer Simulation, 2013, 30(11): 224-213.

[7] Lulu Huang, Hong Ji, Yi Zhu. Analysis of effective working characteristic of the proportional solenoid. 2017 International Conference on Green Energy and Applications, 2017: 35-38.

[8] Qi Zhou, Lijie Wang, Chengwei Wu, et al. Adaptive fuzzy control for nonstrict-feedback systems with input saturation and output constraint. IEEE Transactions on Systems, 2017, 47(1): 1-12.

[9] Shuhong Wang, Xuliang Xiao, Guangyu Xiong. Direct current electromagnets with constant traction characteristic. Journal of Mechanical Engineering, 2008, 44(2): 244-247. (in Chinese) 
[10] Zuoyu Sun, Guoxiu Li, Lan Wang, et al. Effects of structure parameters on the static electromagnetic characteristics of solenoid valve for an electronic unit pump. Energy Conversion and Management, 2016, 113(2016): 119-130.

[11] A Maeck, H Hofmann, A Lorke. Pumping methane out of aquatic sediments - forcing mechanisms that affect the temporal dynamics of ebullition. Biogeosciences Discussions, 2013, 10(11): 18687-18722.

[12] Thompson Company. New solenoid valve from Thompson. World Pumps, 2005, 469: 12-19.

[13] Yuriy Bakhvalov, Valeriy Grechikhin, Oleg Kravchenko, et al. Optimal design of shell-type electromagnets of XY-coordinate electric actuator. 2016 IX International Conference on Power Drives Systems (ICPDS), 2016: 1-4.

[14] Gang Tao, Huiyan Chen. Optimal design of the magnetic field of a highspeed response solenoid valve. Journal of Material Processing Technology, 2002, 129(s1): 555-558.

[15] K Dasgupta, J Watton. Dynamic analysis of proportional solenoid controlled piloted relief valve by bond graph. Simulation Modelling Practice \& Theory, 2005, 13(1): 21-38.

[16] Peng Liu, Liyun Fan, Wei Zhou, et al. Static performance of high-speed electromagnet with permanent magnet based on parallel magnetic circuit. Transactions of CSICE, 2016, 5(34): 449-455.

[17] Qilei Wang, Fengyu Yang, Qian Yang, et al. Experimental analysis of new high-speed powerful digital solenoid valves. Energy Conversion and Management, 2011, 52(5): 2309-2313.

[18] Qiang Cheng, Zhendong Zhang, Hui Guo, et al. Improved processing and performance of GDI injector based on metal injection molding technology. International Journal of Applied Electromagnetics and Mechanics, 2014, 44(1): 99-114.

[19] Qianfeng Liu, Hanliang Bo, Benke Qin. Experimental study and numerical analysis on electromagnetic force of direct action solenoid valve. Nuclear Engineering and Design, 2010, 240(12): 4031-4036.

[20] Songhua Li, Chuang Zuo, Lixiu Zhang, et al. Force output characteristic of piezoelectric actuators. Journal of Shenyang Jianzhu University (Natural Science), 2018, 34(2): 350-358.
[21] Jinhai Gao, Lina Hao, Chaoqun Xiang, et al. Adaptive control of piezoelectric ceramic actuator with composite ADRC. Journal of Northeastern University (Natural Science), 2018, 39(8): 1158-1162.

[22] L Joseph, M L Mcintyre, M Mohebbi. Parameter estimation and a series of nonlinear observers for the system dynamics of a linear vapor compressor. IEEE Transactions on Industrial Electronics, 2016, 63(11): 6736-6744.

[23] Mizuno Takeshi, Hirasawa Yuji, Takasaki Masaya. Self-sensing magnetic suspension using an $\mathrm{H}$-bridge type hystersis amplifier operating in two quadrants. IEEE Trans. Ind. Electron., 2015, 62(6): 3619-3628.

[24] I Dülk, T Kovácsházy. Parameter estimation in linear electromagnetic devices. IEEE Trans. Ind. Electron., 2015, 62(6): 3619-3628.

[25] Qinghui Yuan, Perry Y Li. Self-sensing actuators in electro hydraulic actuators. Proceedings of the 2004 ASME International Mechanical Engineering Congress, Anaheim, CA, USA, No. IMECE2004-6, 2004.

[26] Le Q Minh, M T Pham, M Tavakoli, et al. Bilateral control of nonlinear pneumatic teleoperation system with solenoid valves. IEEE Transactions on Control Systems Technology, 2013, 21(4): 1463-1470.

[27] Ling Zhao, Bin Zhang, Hongjiu Yang, et al. Finite-time tracking control for pneumatic servo system via extended state observer. IET Control Theory \& Applications, 2017, 11(16): 2808-2816.

[28] K Ahn, S Yokota. Intelligent switching control of pneumatic actuator using on/off solenoid valves. Mechatronics, 2002, 15(6): 683-702.

[29] Xiaoqing Xu, Long Quan, Xuping Wang. Principle and characteristics of novel 2-degree-of-freedom electro-mechanical transformers used in valves. Proceedings of the CSEE, 2010, 30(3): 119-124.

[30] Jianhui Zhao, Meiling Wang, Zhangjun Wang, et al. Different boost voltage effects on the dynamic response and energy losses of high-speed solenoid valves. Applied Thermal Engineering, 2017, 123(2017): 1494-1503.

\section{Submit your manuscript to a SpringerOpen ${ }^{\circ}$ journal and benefit from:}

- Convenient online submission

- Rigorous peer review

- Open access: articles freely available online

- High visibility within the field

- Retaining the copyright to your article

Submit your next manuscript at springeropen.com 